
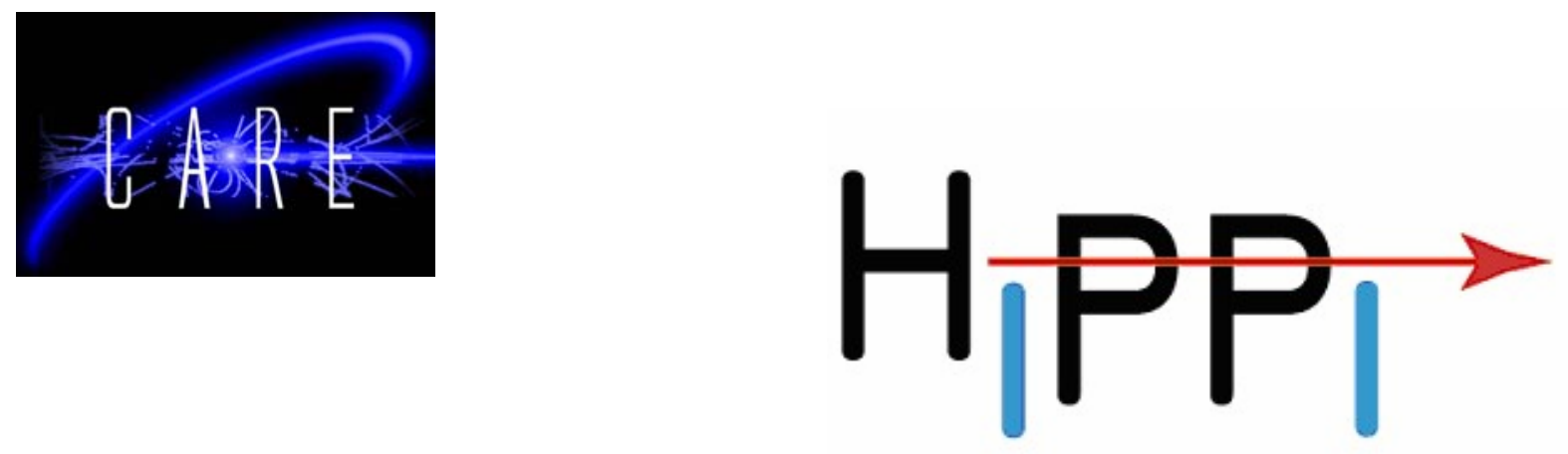

\author{
Heavy Ion High Intensity Upgrade of the GSI UNILAC \\ W. Barth, L. Dahl, M. Galonska, J. Glatz, L. Groening, R. Hollinger, S. Richter, \\ and S. Yaramyshev \\ Gesellschaft für Schwerionenforschung, D-64291 Darmstadt, Germany
}

\begin{abstract}
For the future Facility for Antiproton and Ion Research (FAIR) at Darmstadt the present GSIaccelerator complex, consisting of the linear accelerator UNILAC and the heavy ion synchrotron SIS 18, is foreseen to serve as $\mathrm{U}^{28+}$-injector for up to $10^{12}$ particles/s. After a new High Current Injector (HSI) was installed, many different ion species were accelerated in the UNILAC for physics experiments. In 2001 a high energy physics experiment used up to $2.10^{9}$ uranium ions per SIS 18-spill $\left(\mathrm{U}^{73+}\right)$ while a MEVVA ion source was in routine operation for the first time. In the past two years, different hardware measures and careful fine tuning in all sections of the UNILAC resulted in an increase of the beam intensity to $9.5 \cdot 10^{10} \mathrm{U}^{27+}$-ions per $100 \mu$ s or $1.5 \cdot 10^{10} \mathrm{U}^{73+}$-ions per $100 \mu$ s. The contribution reports results of beam measurements during the high current operation with uranium beams (pulse beam power up to $0.5 \mathrm{MW}$ ). One of the major tasks was to optimize the beam matching to the Alvarez-DTL. In addition further upgrades, including improved beam diagnostics, are described, which allow to fill the SIS 18 up to its space charge limit (SCL) of $2.7 \cdot 10^{11} \mathrm{U}^{28+}$-ions per cycle.
\end{abstract}

Contribution to the Workshop ICFA-HB2004, Bensheim, Germany

Work supported by the European Community-Research Infrastructure Activity under the FP6 "Structuring the European Research Area" programme (CARE, contract number RII3-CT2003-506395). 


\title{
Heavy Ion High Intensity Upgrade of the GSI UNILAC
}

\author{
W. Barth, L. Dahl, M. Galonska, J. Glatz, L. Groening, R. Hollinger, S. Richter, \\ and S. Yaramyshev ${ }^{1}$
}

Gesellschaft für Schwerionenforschung, D-64291 Darmstadt, Germany

\begin{abstract}
For the future Facility for Antiproton and Ion Research (FAIR) at Darmstadt the present GSI-accelerator complex, consisting of the linear accelerator UNILAC and the heavy ion synchrotron SIS 18, is foreseen to serve as $\mathrm{U}^{28+}$-injector for up to $10^{12}$ particles/s. After a new High Current Injector (HSI) was installed, many different ion species were accelerated in the UNILAC for physics experiments. In 2001 a high energy physics experiment used up to $2 \cdot 10^{9}$ uranium ions per SIS 18 -spill $\left(\mathrm{U}^{73+}\right)$ while a MEVVA ion source was in routine operation for the first time. In the past two years, different hardware measures and careful fine tuning in all sections of the UNILAC resulted in an increase of the beam intensity to $9.5 \cdot 10^{10} \mathrm{U}^{27+}$-ions per $100 \mu \mathrm{s}$ or $1.5 \cdot 10^{10} \mathrm{U}^{73+}$-ions per $100 \mu \mathrm{s}$. The contribution reports results of beam measurements during the high current operation with uranium beams (pulse beam power up to $0.5 \mathrm{MW}$ ). One of the major tasks was to optimize the beam matching to the Alvarez-DTL. In addition further upgrades, including improved beam diagnostics, are described, which allow to fill the SIS 18 up to its space charge limit (SCL) of $2 \cdot 7 \cdot 10^{11} \mathrm{U}^{28+}$-ions per cycle.
\end{abstract}

Keywords: Ion sources, Heavy ions, Beam focusing, Matching, Emittance growth

PACS: 07.77.Ka, 41.75.Ak., 41.85.Ew, 41.85.Lc

\section{INTRODUCTION}

The conceptual design of the Facility for Antiprotons and Ion Research (FAIR) at GSI in Darmstadt (Fig. 1) has evolved from the science requirements: higher intensities will be achieved compared to the present GSI accelerator facility, through faster cycling and, for heavy ions, lower charge state which enters quadratically into the Space Charge Limit (SCL). The desired energy of up to $1.5 \mathrm{GeV} / \mathrm{u}$ for radioactive beam production is delivered by the synchrotron SIS 100, which also generates intense beams of energetic protons up to $30 \mathrm{GeV}$ for pbar-production. The energy of $30 \mathrm{GeV} / \mathrm{u}$ for heavy ions is generated by using higher charge states in combination with the slower cycling synchrotron SIS 300. The SIS 300 can also be used as a stretcher for radioactive beams, which can be injected, cooled, and stored in a system of rings with internal targets and in-ring experimentation. The various rings may be shared for the use with different beams. The present GSI accelerator complex will serve as an injector for high intensity heavy ions to fill the

\footnotetext{
${ }^{1}$ on leave from ITEP, Moscow, Russia
}

SIS 100 to its space charge limit. In the last three years GSI put effort in increasing the delivered uranium intensities to the SIS 18. An additional upgrade program for the UNILAC is foreseen to reach the required beam brilliance until 2009. For uranium

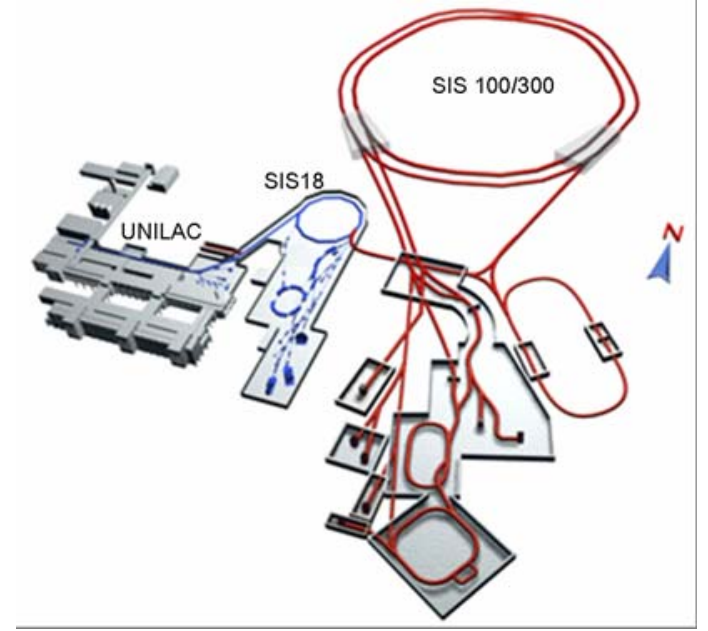

FIGURE 1. The proposed Accelerator facility FAIR at GSI in Darmstadt [1]. 
Table 1. Design uranium beam parameters at UNILAC and SIS 18 injection [2].

\begin{tabular}{|c|c|c|c|c|}
\hline & $\begin{array}{c}\text { HSI } \\
\text { exit }\end{array}$ & $\begin{array}{c}\text { Alvarez } \\
\text { entrance }\end{array}$ & $\begin{array}{c}\text { SIS 18 } \\
\text { injection }\end{array}$ & $\begin{array}{c}\text { Required } \\
\text { for FAIR }\end{array}$ \\
\hline Ion species & ${ }^{238} \mathrm{U}^{4+}$ & ${ }^{238} \mathrm{U}^{28+}$ & ${ }^{238} \mathrm{U}^{73+}$ & ${ }^{238} \mathrm{U}^{28+}$ \\
\hline El. Current [mA] & 15 & 12.5 & 4.6 & 15.0 \\
\hline Part. per $100 \mu \mathrm{s}$ pulse & $2.3 \cdot 10^{12}$ & $2.8 \cdot 10^{11}$ & $4.2 \cdot 10^{10}$ & $3.3 \cdot 10^{11}$ \\
\hline Energy [MeV/u] & 1.4 & 1.4 & 11.4 & 11.4 \\
\hline$\Delta \mathrm{W} / \mathrm{W}$ & $4 \cdot 10^{-3}$ & $\pm 1 \cdot 10^{-2}$ & $\pm 2 \cdot 10^{-3}$ & $\pm 2 \cdot 10^{-3}$ \\
\hline$\varepsilon_{\mathrm{n}, \mathrm{x}}[\mathrm{mm} \mathrm{mrad}]$ & 0.5 & 0.75 & 0.8 & 0.8 \\
\hline$\varepsilon_{\mathrm{n}, \mathrm{y}}[\mathrm{mm} \mathrm{mrad}]$ & 0.5 & 0.75 & 2.5 & 2.5 \\
\hline
\end{tabular}

(reference ion) the UNILAC has to deliver $3.3 \cdot 10^{11}$ $\mathrm{U}^{28+}$-particles per $100 \mu$ s (see Table 1 ) to the present synchrotron SIS 18 . Currently for a $15 \mathrm{emA}{ }^{238} \mathrm{U}^{4+}$ beam from the HSI [4] up to $4 \cdot 10^{10} \mathrm{U}^{73+}$ particles should be delivered to the SIS 18 (during $100 \mu \mathrm{s}$ ), while the SIS 18-SCL is reached by a 20 turn injection into the horizontal phase space. The HSI-front end consists of ion sources of MEVVA-, MUCIS- or Penning-type and a low energy beam transport system (LEBT). The $36 \mathrm{MHz}$ IH-RFQ accelerates the ion beam from $2.2 \mathrm{keV} / \mathrm{u}$ to $120 \mathrm{keV} / \mathrm{u}$. In the following short 11 cell adapter RFQ (Super Lens) the beam is matched to the IH-DTL, consisting of two separate tanks accelerating the beam up to the final HSI-energy of $1.4 \mathrm{MeV} / \mathrm{u}$. The HSI-beam is stripped and one charge state is selected (e.g. 28+ for uranium beams). In the Alvarez DTL the high intensity HSI beam is accelerated without significant particle loss. The transfer line (TK) to the SIS 18 includes a foil stripper and another charge state separator system.

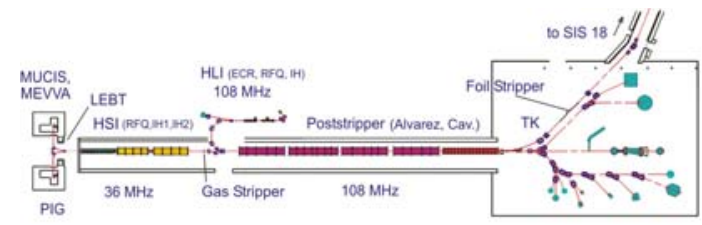

FIGURE. 2. Schematic overview of the GSI UNILAC [3].

\section{HSI UPGRADE}

For the requested operation with intense uranium beams the performance of the UNILAC was significantly improved [5]: the MEVVA ion source was renewed and the new MEVVA type source VARIS was developed. The operation life is typically 7 days. The pulse to pulse stability was enhanced resulting in high beam availability. A fraction of $67 \%$ of $\mathrm{U}^{4+}$ was reached. Enhancement of the extraction system resulted in a higher field strength $(11 \mathrm{kV} / \mathrm{mm})$.
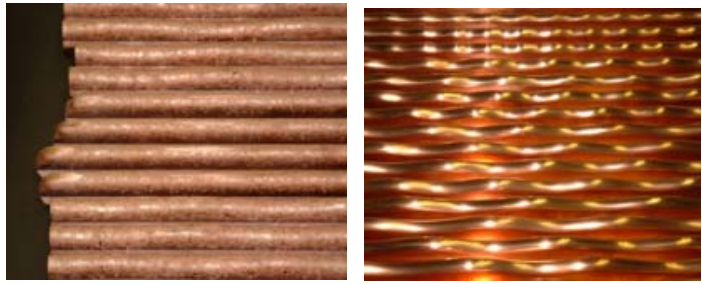

FIGURE 3. Upgrade of the HSI-RFQ; RFQ-electrodes after five years of operation (left), new electrodes before assembly (right).

The total emission current is $170 \mathrm{~mA} / \mathrm{cm}^{2}$ [6].

Due to the high rf surface field in the RFQ and in the Super Lens, rf-conditioning has to be performed if low charged heavy ions must be accelerated in the HSI. Permanent rf-conditioning with a low duty factor (approx. $0.3 \%$ ) in a time sharing mode with the regular beam time allows for the required rfamplitudes. In 2002 the Super Lens was completely

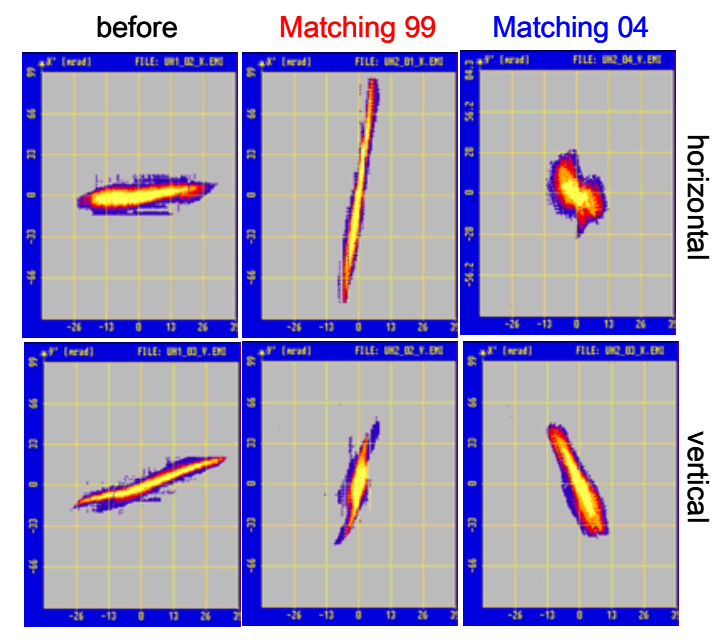

FIGURE 4. Measured $\mathrm{U}^{4+}$ emittance before and after the quadrupole quartet (left) for the two matching cases. The transmission for the new matching (2004) is $15 \%$ higher.

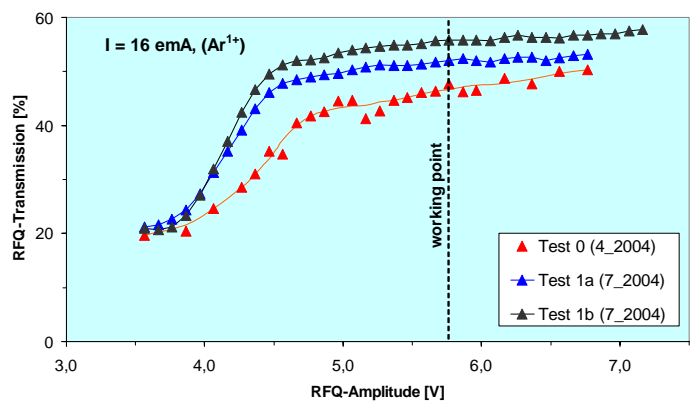

FIGURE 5. Measured transmission for a high current argon beam before and after the upgrade of the RFQelectrodes. 
dismounted. In an upgrade scheme the rf-performance was significantly improved: the maximum surface field strength was slightly decreased, the surface quality was improved and a new plunger design was applied [7].

Since the end of May 2004 a comparable upgrade measure for the RFQ was in progress. In Fig. 3 (left) some of the dismantled RFQ-electrodes are shown. After five years of operation the electrode surface was damaged along the whole structure, requiring significantly higher input rf-power during operation at the design limit. The electrodes were reproduced; additional copper plating should lead to a reduction of "dark current" contributions during high power operation.

The RFQ input radial matcher (IRM) was redesigned to improve the matching to the RFQ. The new matching leads to a smaller beam diameter in the quadrupole channel, resulting in improved particle transmission. While the RFQ was de-installed for the proposed upgrade measures, the matching was investigated using beam transformers and emittance measurement devices before and after the quadrupole quartet in the LEBT-section. As predicted by simulations with the DYNAMION-code, based on high current uranium emittance measurements, the measured gain in particle number is close to $15 \%$ (see Fig. 4). During recomissioning of the RFQ a high intensity $\mathrm{Ar}^{1+}$ beam was used. In Fig. 5 the measured beam transmission as a function of the rf-voltage is presented together with the data before the upgrade. At the working point the expected gain in RFQtransmission is verified. The achieved maximum Arintensity (after RFQ) is $10.6 \mathrm{emA}$, being above the design limit (10 emA).

After a heavy sparking inside the IH1-structure of the HSI at the beginning of 2003 an inner triplet lens was substituted. The new advanced design provides for an improved electromagnetic performance and the outer walls of the big drift tube are directly watercooled now. As shown in Fig. 6 the geometry of the

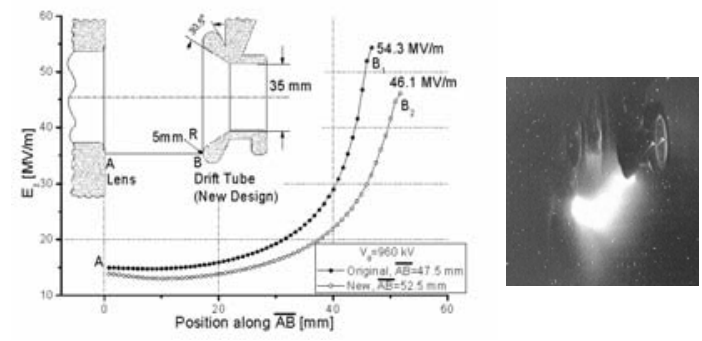

FIGURE 6. Maximum electric field between the first big drift tube (housing a triplet lense) and the subsequent small drift tube (left); before the improved drift tube geometry was applied (right). subsequent small drift tube was slightly changed, leading to a different field distribution $\left(\mathrm{E}_{\mathrm{z}}\right)$ and lower "dark current" contributions during operating at the highest rf-power level [8].

\section{GASSTRIPPER PERFORMANCE}

With the reduction of the available apertures in the stripper box, it was possible to increase the stripper gas density by $50 \%$. For medium intense uranium beams (6.5 emA, $\mathrm{U}^{4+}$ ) this leads to the expected gain for the desired charge state 28+ (up to $12.8 \%$ ). The desired equilibrium charge state distribution is reached for a $70 \%$ higher gas density. Currently the power of the vacuum pumps is not sufficient to compensate this gas load. Due to a temporary increase of the gas density by $70 \%$ the measured total uranium current (all charge states) was increased to $41 \mathrm{emA}$. The defocusing effect of the space charge forces leads to particle loss in the transport section after the stripping area. This can be compensated by increasing the transverse beam spot in the space charge dominated beam transport. The high stripper gas density for the necessary enlarged apertures has to be provided by an enhanced vacuum pumping speed. For the proposed higher uranium intensities the present stripper section is not sufficient to meet the FAIR-requirements and has to be redesigned.

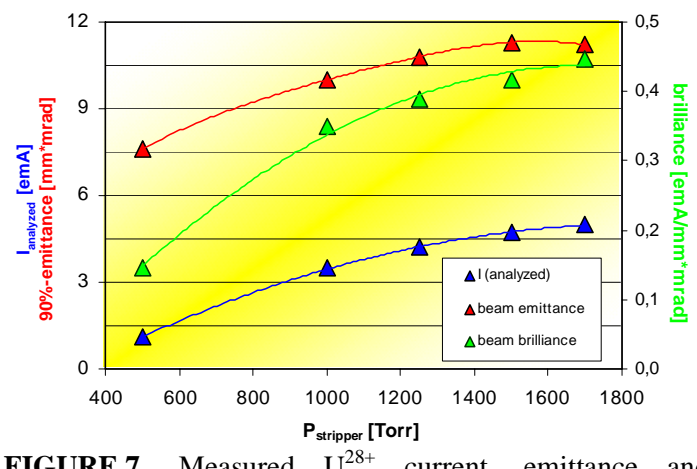

FIGURE 7. Measured $\mathrm{U}^{28+}$ current, emittance and brilliance after charge state separation as a function of the stripper gas density; the input $\mathrm{U}^{28+}$-current is $6 \mathrm{emA}$.

\section{BEAM MATCHING TO THE ALVAREZ}

Usually empirical matching can be done by variation of the quadrupole settings preceding the DTL until a sufficient transmission through the Alvarez 


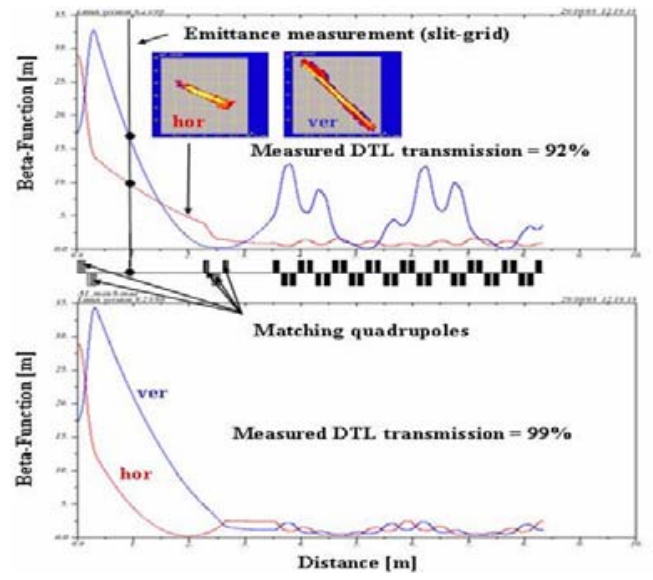

FIGURE 8.Systematic matching of the Twiss parameters to the periodic solution of the first Alvarez tank.

section of more than $90 \%$ is achieved. In order to increase the transmission close to $100 \%$, a systematic matching procedure was proposed and realized during machine experiments. In general the beam is mismatched with an enlarged beta function oscillation along the Alvarez accelerator, which may cause transmission losses and emittance growth. Using the quadrupole settings in the first cells of the DTL the periodic solution for the Alvarez tank is calculated. To match the periodic DTL solution, a fitting routine involving the five matching quadrupoles (Fig. 8) is applied. For the highest available uranium intensity the losses along the Alvarez section are reduced from $8 \%$ to less than $1 \%$ [9].

\section{HIGH CURRENT DTL PERFORMANCE}

The postaccelerator performance can also be improved by the optimization of the quadrupole settings in the Alvarez DTL, being most important in the $1^{\text {st }}$ Alvarez tank. Due to the high mass over charge ratio $(\mathrm{m} / \zeta)$ of ${ }^{238} \mathrm{U}^{28+}$ ions, the maximum zero current phase advance $\sigma_{0}$ in the Alvarez DTL is limited to $45^{\circ}$ mainly by the quadrupole power supplies. For a ${ }^{40} \mathrm{Ar}^{10^{+}}$ beam the $\mathrm{m} / \zeta$ is lower; so that the phase advance influence on the transmission in the Alvarez DTL can be investigated at an extended range of $\sigma_{0}$-values. As shown in Fig. 9, a phase advance $\sigma_{0}>50^{\circ}$ is required for an improved beam brilliance for the SIS 18injection.

A bottleneck behind the Alvarez section was eliminated by the reduction of the number of the Single Gap Resonators from 15 to 10 - allowing beam transport with smaller beta-function modulation and better transmission. Adjustments along all sections of the UNILAC were performed additionally with high accuracy.

\section{IMPROVED TRANSFER TO THE SIS 18}

The optimized matching of space charged dominated ion beams is essential for a loss free injection into the SIS 18. Transverse beam emittance measurements at different positions along the TK were done. In particular, different foil stripping modes were investigated. For the high current heavy ion beam operation e.g. a sweeping mode is routinely applied to minimize the thermal stress of the carbon stripper foil. A longitudinal emittance measurement set-up was commissioned at the entrance to the TK. It is used extensively to tune the rebuncher cavities in the UNILAC. In addition, a test bench is in use for measurements of longitudinal bunch profiles, which enables the investigation of the final debunching to

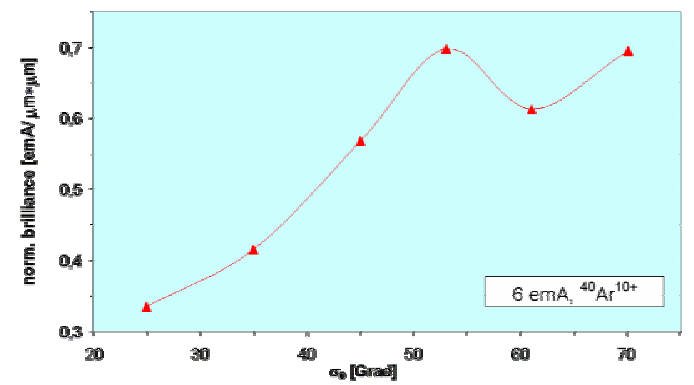

FIGURE 9. Normalized beam brilliance for a high current argon beam after the $4^{\text {th }}$ Alvarez-tank as a function of $\sigma_{0}$.

SIS 18. Multi particle simulations by means of the PARMILA-code were performed allowing for a detailed analysis of experimental results for different ion currents [10].

\section{${ }^{238}$ U INTENSITIES FOR THE SIS 18}

Table 2. Uranium beam intensities

\begin{tabular}{|c|c|c|c|}
\hline & $\zeta$ & Dec 01 & Dec 03 \\
\hline LEBT & $4+$ & 7,5 & 11,7 \\
\hline HSI & $4+$ & 3,75 & 6,4 \\
\hline gas stripper & $4+$ & 2,5 & 5,3 \\
\hline Alvarez & $28+$ & 1,8 & 4,8 \\
\hline Single Gap Resonators & $28+$ & 1,17 & 4,8 \\
\hline Foil Stripper & $28+$ & 0,94 & 4,5 \\
\hline SIS 18-Injection & $73+$ & 0,3 & 2.0 \\
\hline
\end{tabular}

In Fig. 10 the achieved uranium intensities in the UNILAC and TK are summarized (2001-2003), reached by the measures mentioned above and by an 
extended experimental program dedicated to improve the overall UNILAC performance for heavy ion high current operation. In December 2003 an $\mathrm{U}^{73+}$ intensity of $2.0 \mathrm{emA}(27.5 \mathrm{p \mu A})$ was reached for the first time

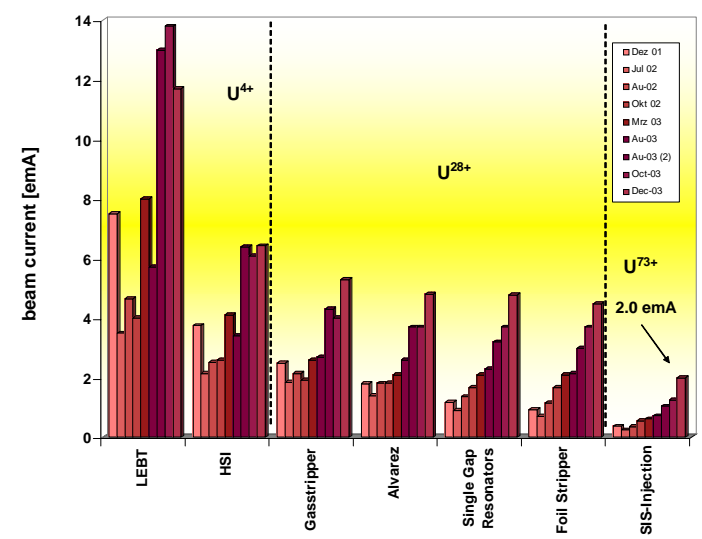

FIGURE 10. Improvement of the UNILAC-uranium beam intensities during the last three years.

at the injection to SIS 18, which corresponds to $1.7 \cdot 10^{10}$ particles per $100 \mu \mathrm{s}$. Before foil stripping $4.5 \mathrm{emA}(160 \mathrm{p \mu A})$ of $\mathrm{U}^{28+}$ beam intensity was achieved $\left(1.0 \cdot 10^{11}\right.$ particles per $\left.100 \mu \mathrm{s}\right)$. The optimized total particle transmission for high current uranium beams through HSI, stripper section, Alvarez DTL, Single Gap Resonator chain, and TK is $50 \%$, if the particle losses during charge separation after the two strippers are taken into account. Compared to the design transmission (90\%) the lack is mainly caused by a bottleneck in the front end area of the HSI-linac.

\section{CONCLUSION AND OUTLOOK}

An extended upgrade program in the UNILAC and TK in combination with machine investigations resulted in a seven times higher uranium beam intensity offered for the injection into the synchrotron SIS 18. Mainly the improved ion source performance, an upgrade of the HSI-structures, the increased stripper gas density, the optimization of the Alvarez-matching, the reduction in the Single Gap Resonator section, the applied sweeper mode for foil stripper operation, and the use of various newly developed beam diagnostics devices comprised the successful development program. The UNILAC-upgrade already started will be continued with the investigation of a new front end for $\mathrm{U}^{4+}$ [11], stronger power supplies for the Alvarez quadrupoles, a charge state separator system [12] in the foil stripper section and versatile (partly nondestructive) beam diagnostics devices [13], sufficient for the operation with megawatt heavy ion beams.
We acknowledge the support of the European Community-Research Infrastructure Activity under the FP6 "Structuring the European Research Area" program (CARE, contract number RII3-CT-2003506395) and INTAS (project 03-54-3543).

\section{REFERENCES}

1. W. Henning, An International Accelerator Facility for Research with Ions and Antiprotons, EPAC2004, Luzern, Switzerland, to be published.

2. W. Barth, et al., Achievements of the High Current Beam Performance of the GSI UNILAC, EPAC2004, Luzern, Switzerland, to be published.

3. W. Barth, e. al., Commissioning of the $1.4 \mathrm{MeV} / \mathrm{u}$ High Current Heavy Ion Linac at GSI, LINAC2000, Monterey, U.S.A., p. 1033 (2000).

4. U. Ratzinger et al., , The New GSI Prestripper Linac for High Current Heavy Ion Beams, LINAC96, Geneva, Switzerland, p. 288 (1996).

5. R. Hollinger, et al., Beam Emittance Measurements at the HSI at GSI, Proc. of the 9th ICIS-conference, Oakland, U.S.A., p. 1024 (2001).

6. R. Hollinger et al., Development of a vacuum ion source for injection of high current uranium ion beam into the UNILAC at GSI, Rev. Sci. Instrum. 75 (5) (2004), 1595.

7. W. Barth, Experience during Operation with High Current U4+ Beams in the new HSI, LINAC2002, Gyeongju, Korea, p. 347 (2002).

8. W. Barth, et. al., GSI-an. rep. p. 207 (2003).

9. S. Yaramishev, et. al., Investigation of the Beam Matching to the GSI-Alvarez DTL under Space Charge Conditions, LINAC2004, Lübeck, Germany, to be published.

10. S.Richter et al., High current Beam Transport to SIS 18, LINAC2004, Lübeck, Germany, to be published.

11. L. Dahl, et. al., Transport and Injection of heavy ion Beams with High Brilliance for the GSI-HSI, LINAC2002, Gyeongju, Korea, p. 350 (2002).

12. J. Glatz. B. Langenbeck, The High Current Charge Stripper, Charge Separator, and their Magnets for the Beam Transfer line to the Heavy Ion Synchrotron SIS, Conf. on Magnet Technology, IEEE Transactions on Magnetics (2000).

13. W. Barth, et. al, Application of Beam Diagnostics for Intense Heavy Ion Beams at the GSI UNILAC, DIPAC 2003, Mainz, Germany, p. 161 (2003). 